SUBJECT AREAS:

SPECTROSCOPY

OPTICAL METROLOGY

FREQUENCY COMBS

Received

26 November 2013

Accepted

2 January 2014

Published

22 January 2014

Correspondence and requests for materials should be addressed to

T.Y. (yasui.takeshi@ tokushima-u.ac.jp) \section{comb-mode-resolved spectroscopy using swept dual terahertz combs}

\author{
Yi-Da Hsieh', Yuki lyonaga' ', Yoshiyuki Sakaguchi' ', Shuko Yokoyama ',2, Hajime Inaba ${ }^{3,4}$, \\ Kaoru Minoshima ${ }^{4,5}$, Francis Hindle ${ }^{6}$, Tsutomu Araki' \& Takeshi Yasui ${ }^{1,4,7}$
}

\begin{abstract}
${ }^{1}$ Graduate School of Engineering Science, Osaka University, 1-3 Machikaneyama, Toyonaka, Osaka 560-8531, Japan, ${ }^{2}$ Micro Optics Co., Ltd, 2-20, Oe-Nakayama, Nishikyo, Kyoto 610-1 104, Japan, ${ }^{3}$ National Metrology Institute of Japan, National Institute of Advanced Industrial Science and Technology, 1-1-1 Umezono, Tsukuba, Ibaraki 305-8563, Japan, ${ }^{4}$ ERATO Intelligent Optical Synthesizer Project, JST, 1-5-1, Chofugaoka, Chofu, Tokyo 182-8585, Japan, ${ }^{5}$ Graduate School of Informatics and Engineering, The University of Electro-Communications, 1-5-1, Chofugaoka, Chofu, Tokyo 182-8585, Japan, 'Laboratoire de Physico-Chimie de l'Atmosphère, Université du Littoral Côte d'Opale, 189A Av. Maurice Schumann, Dunkerque 59140, France, ${ }^{7}$ Institute of Technology and Science, The University of Tokushima, 2-1 Minami-Josanjima, Tokushima, Tokushima 770-8506, Japan.
\end{abstract}

Optical frequency combs are innovative tools for broadband spectroscopy because a series of comb modes can serve as frequency markers that are traceable to a microwave frequency standard. However, a mode distribution that is too discrete limits the spectral sampling interval to the mode frequency spacing even though individual mode linewidth is sufficiently narrow. Here, using a combination of a spectral interleaving and dual-comb spectroscopy in the terahertz $(\mathrm{THz})$ region, we achieved a spectral sampling interval equal to the mode linewidth rather than the mode spacing. The spectrally interleaved $\mathrm{THz}$ comb was realized by sweeping the laser repetition frequency and interleaving additional frequency marks. In low-pressure gas spectroscopy, we achieved an improved spectral sampling density of $2.5 \mathrm{MHz}$ and enhanced spectral accuracy of $8.39 \times 10^{-7}$ in the THz region. The proposed method is a powerful tool for simultaneously achieving high resolution, high accuracy, and broad spectral coverage in THz spectroscopy.

U se of optical frequency combs has emerged as a new technique for broadband spectroscopy because they have the potential to achieve high spectral resolution in a broad spectrum in addition to high accuracy frequency markers ${ }^{1-3}$. Optical comb has been usually used in combination with a Michelson-interferometer-based Fourier-transform spectrometer (FTS) ${ }^{4}$. However, the spectral resolution in usual FTSs is insufficient to spectrally resolve each mode because the modes are distributed too densely for FTS.

Recently, dual-comb spectroscopy has unlocked further potential for optical-comb-based broadband spectroscopy $^{5-12}$. In this method, one optical comb that contains the measurement information (frequency interval $=$ $f_{\text {rep } 1}$ ) is read with another optical comb with a slight frequency offset (frequency interval $=f_{\text {rep } 2}=f_{\text {rep } 1}+f_{\text {offset }}$ ). This results in the generation of radio-frequency (RF) comb (frequency interval $=f_{\text {offset }}$ ). Since the RF comb is essentially a replica of the measurement comb, downscaled in frequency by the ratio $f_{\text {rep } 1} / f_{\text {offset }}$, one can utilize the comb-mode-resolved spectrum via direct observation of the RF comb. Since the first experimental demonstration of the dual-comb spectroscopy in mid-infrared region $^{6}$ and the following observation of the comb-mode-resolved spectrum in terahertz $(\mathrm{THz})$ region ${ }^{7}$, the implementation of dual-comb spectroscopy has made notable progress in various wavelength regions because of high sensitivity, rapid data acquisition, high spectral resolution, and excellent accuracy ${ }^{8-12}$.

The optical comb mode enables us to achieve a frequency resolution equal to the comb mode linewidth (typically, less than $1 \mathrm{MHz}$ ) when the absolute frequency is determined based on a single comb mode. However, when the comb-mode-resolved spectrum obtained by dual-comb spectroscopy is used for broadband spectroscopy, the spectral sampling interval is limited to the comb mode spacing (typically, from several tens to a few hundred $\mathrm{MHz}$ ) due to the excessively discrete mode distribution. If the comb mode gap is filled by interleaving additional frequency marks, the spectral sampling density and accuracy would be vastly enhanced in the broadband spectroscopy. The combination of such interleaving with dual-comb spectroscopy has been demonstrated in optical region ${ }^{13,14}$ and sub-Doppler spectroscopy has now been achieved by making full use of resolution improvement through interleaving ${ }^{15,16}$. However, there have been no attempts to interleave $\mathrm{THz}$ comb. 
In this article, we propose a spectrally interleaved dual- $\mathrm{THz}$ comb spectroscopy based on a swept dual-THz-comb technique. Furthermore, the proposed method was effectively applied to highresolution, high-accuracy, broadband spectroscopy of gas-phase molecules at low pressure.

\section{Results}

Principle of operation. We first describe spectral behavior of dual$\mathrm{THz}$-comb spectroscopy in optical, $\mathrm{THz}$, and $\mathrm{RF}$ regions as shown in Fig. 1(a) ${ }^{7}$. When the first optical comb (frequency interval $=f_{\text {rep } 1}$ ) is incident onto an electrically-biased photoconductive antenna for $\mathrm{THz}$ emission (PCA emitter), it is down-converted to the $\mathrm{THz}$ region without any change to the frequency spacing because the PCA emitter squares the electric field of the optical comb via the quadratic detection, converts the optical intensity to electric carriers, and emits a frequency comb of electromagnetic $\mathrm{THz}$ wave $(\mathrm{EM}-\mathrm{THz}$ comb) via the dipole radiation. The EM- $\mathrm{THz}$ comb is a harmonic frequency comb of $f_{\text {rep } 1}$ without a frequency offset (freq. $=f_{\text {rep } 1}$, $\left.2 f_{\text {rep } 1}, 3 f_{\text {rep } 1}, \cdots \cdots \bullet \bullet, n f_{\text {rep } 1}\right)$. Next, we consider what happens when the second optical comb (frequency interval $=f_{\text {rep } 2}=f_{\text {rep } 1}+f_{\text {offset }}$ ) slightly detuned from the first one is incident onto a non-biased, photoconductive antenna for $\mathrm{THz}$ detection (PCA detector). In this case, $\mathrm{THz}$ comb of photocarrier with frequency spacing of $f_{\text {rep } 2}$ [PC-THz comb; freq. $=f_{\text {rep } 2}, 2 f_{\text {rep } 2}, 3 f_{\text {rep } 2}, \cdots \cdots \bullet, n f_{\text {rep } 2}=\left(f_{\text {rep } 1}+\right.$ $\left.\left.f_{\text {offset }}\right), 2\left(f_{\text {rep } 1}+f_{\text {offset }}\right), 3\left(f_{\text {rep } 1}+f_{\text {offset }}\right), \cdots \cdots \cdots, n\left(f_{\text {rep } 1}+f_{\text {offset }}\right)\right]$ is induced in PCA detector. Detection of EM-THz comb with the PCA detector including the $\mathrm{PC}-\mathrm{THz}$ comb allows us to detect the $\mathrm{RF}$ comb (frequency interval $=f_{\text {offset }}$ ) as a result of the multifrequency-heterodyning photoconductive mixing occurring between the EM-THz and PC-THz combs.

Frequency-domain signal acquisition using an RF spectrum analyzer enables a direct observation of comb-mode-resolved spectrum in RF comb ${ }^{7}$. However, signal acquisition efficiency of RF comb is limited by super-heterodyne technique used in RF spectrum analyzer because it acquires only the single frequency-component signal coinciding with the frequency of its local oscillator at any given moment and then obtains the overall spectrum by sweeping the frequency of the local oscillator. On the other hand, a combination of timedomain signal acquisition with Fourier transformation (FT) improves signal acquisition efficiency because it can capture all frequency components of the RF comb at once in the time domain. To obtain the THz-comb-mode-resolved spectrum at better signal-tonoise ratio and dynamic range, we used an asynchronous-opticalsampling $\mathrm{THz}$ time-domain spectroscopy (ASOPS-THz-TDS) system consisting of two mode-locked lasers with slightly mismatched repetition frequencies ${ }^{11,17-20}$. Although the ASOPS-THzTDS has been usually used for acquiring a temporal waveform of a single $\mathrm{THz}$ pulse within one pulse period $\left(=1 / f_{\text {rep } 1}\right)$, the non-mechanical nature of the time-delay scanning enables us to extend the time window $t_{w}$ up to multiple pulse periods $\left(=N_{p} / f_{\text {rep } 1}\right.$, where $N_{p}$ is number of $\mathrm{THz}$ pulse) in order to acquire the temporal waveform of a THz pulse train, as shown in Fig. 1(b). Taking FT of the temporal waveform gives the detailed spectrum of the $\mathrm{THz}$ comb modes because FT of the periodical THz pulses imprints frequency modulation on the broadband $\mathrm{THz}$ spectrum. We call it a standard $\mathrm{THz}$ comb. In this case, frequency spacing between comb modes is equal to $f_{\text {rep } 1}$ whereas linewidth of the comb mode is determined by $1 / t_{w}$, namely, $f_{\text {rep } 1} / N_{p}$.

We should consider how we could interleave the additional marks into the gap produced between the comb modes of a standard $\mathrm{THz}$ comb. The $\mathrm{THz}$ comb is a harmonic frequency comb of the laser repetition frequency $f_{\text {rep } 1}$ and does not contain a carrier-envelopeoffset frequency $f_{\text {ceo }}$. Therefore, the absolute frequency of each comb mode ( $=m f_{\text {rep } 1}$, where $m$ is the order of $\mathrm{THz}$ comb modes) can be tuned by changing $f_{\text {rep } 1}$. For example, since a single sweep of $f_{\text {rep } 1}$ by $\delta f_{\text {rep } 1}$ causes a frequency shift of $\mathrm{m}$-order mode (freq. $=m f_{\text {rep } 1}$ ) by $m \delta f_{\text {rep } 1}$, M-times repetition of this sweep will lead to a total shift of $\mathrm{M} m \delta f_{\text {rep } 1}$ in $m f_{\text {rep } 1}$. If incremental sweeping of the comb mode is repeated at an interval equal to the mode linewidth, namely, $m \delta f_{\text {rep } 1}$ $=f_{\text {rep } 1} / N_{p}$ or $\delta f_{\text {rep } 1}=f_{\text {rep } 1} /\left(m N_{p}\right)$, and all of the resulting comb spectra are overlaid in the spectral domain, as shown in Fig. 1(c), the frequency gaps of a standard comb can be completely removed. To fill the frequency gaps, such incremental sweeping has to be repeated times of $\mathrm{M}\left[=f_{\text {rep } 1} /\left(m \delta f_{\text {rep } 1}\right)=N_{p}\right]$. In this way, a spectrally interleaved $\mathrm{THz}$ comb will be achieved. This is equivalent to continuous sweeping of a single-mode, narrow-linewidth $\mathrm{CW}-\mathrm{THz}$ wave. The resulting spectral resolution will be equal to the linewidth of the comb mode. It is important to note that $M$ is sufficiently large to fully interleave the comb gap because the tunable range of $f_{\text {rep } 1}$ is within $1 \%$ of $f_{\text {rep } 1}$ at maximum.

Figure 2 illustrates a schematic diagram of the experimental setup, which contains dual mode-locked Er-doped fibre lasers (ASOPS TWIN 250 with P250, Menlo Systems; centre wavelength $\lambda_{c}=$ $1550 \mathrm{~nm}$, pulse duration $\Delta \tau=50 \mathrm{fs}$, mean power $P_{\text {mean }}=$ $500 \mathrm{~mW}, f_{\text {rep } 1}=250,000,000 \mathrm{~Hz}, f_{\text {rep } 2}=250,000,050 \mathrm{~Hz}$, and $f_{\text {offset }}$ $=50 \mathrm{~Hz}$ ) and a THz optical setup for low-pressure gas spectroscopy $^{12}$. The temporal waveform of the pulse train of the THz electric field was acquired at a sampling interval of $100 \mathrm{fs}$. Finally, the

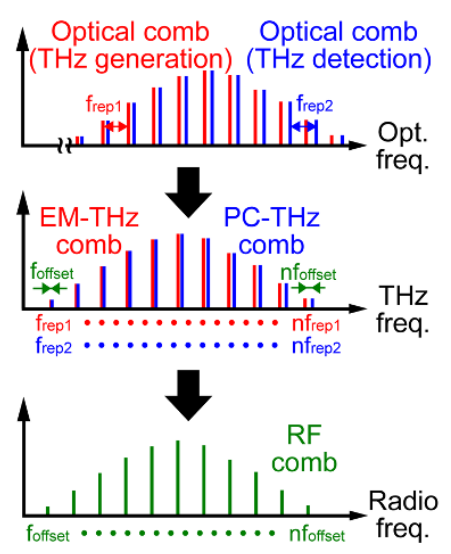

(a)

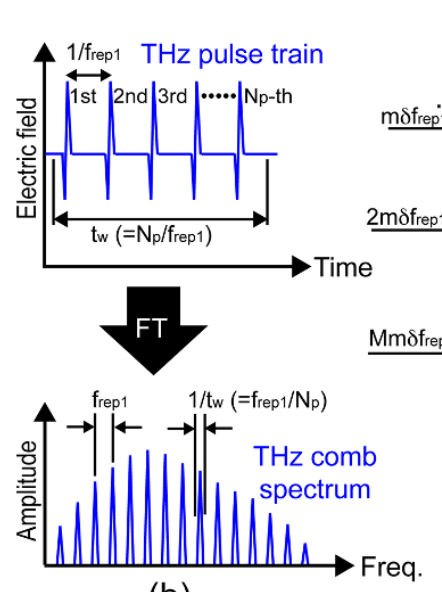

(b)

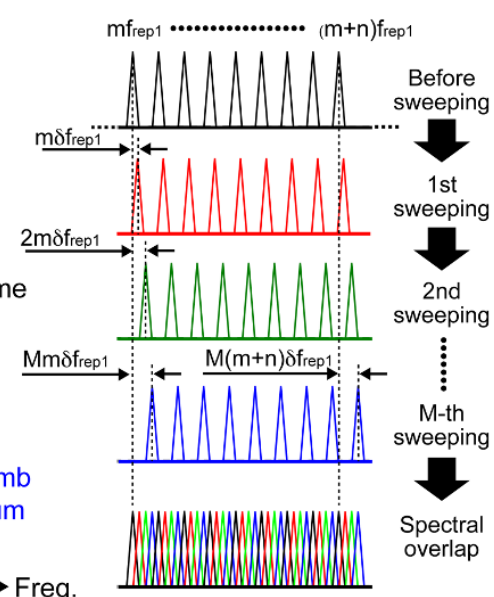

(c)

Figure 1 | (a) Spectral behavior of dual-THz-comb spectroscopy in optical, THz, and RF regions. (b) Temporal waveform of THz pulse train and corresponding $\mathrm{THz}$ comb spectrum. (c) Spectrally interleaved $\mathrm{THz}$ comb achieved by incremental sweeping of $\mathrm{THz}$ comb mode and spectral overlapping. 


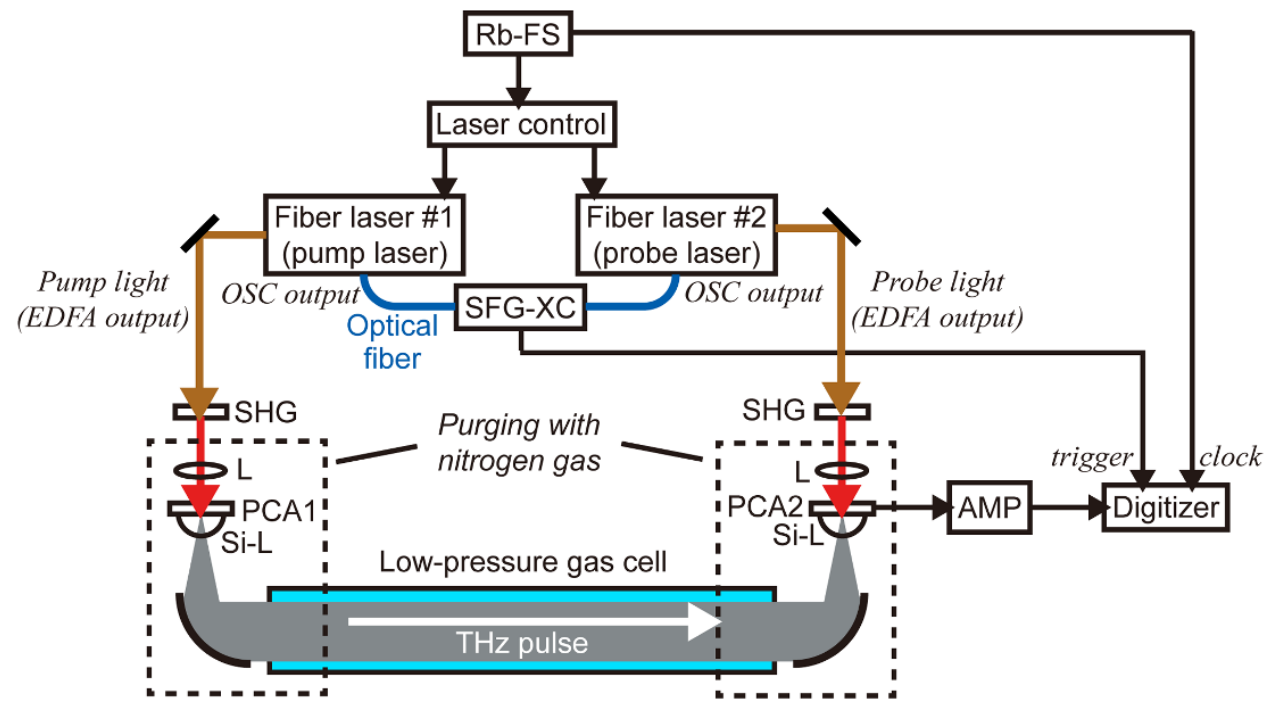

Figure $2 \mid$ Experimental setup. Rb-FS, rubidium frequency standard; SFG-XC, sum-frequency-generation cross-correlator; SHG, second-harmonicgeneration crystals; L, lenses; EDFA, erbium-doped fibre amplifier; OSC, erbium-doped fibre oscillator; PCA1, dipole-shaped low-temperature-GaAs photoconductive antenna for THz emitter; PCA2, dipole-shaped low-temperature-GaAs photoconductive antenna for THz detector; Si-L, silicon lenses; AMP, current preamplifier.

amplitude spectrum of the $\mathrm{THz}$ comb was obtained by $\mathrm{FT}$ of the temporal waveform of the $\mathrm{THz}$ pulse train.

Spectroscopy of water vapour. Since water vapour plays an important role in the Earth system, particularly in the radiation budget, it is important to measure it correctly from the viewpoint of global warming ${ }^{21}$. Here, the rotational transition $1_{10} \leftarrow 1_{01}$ at $0.557 \mathrm{THz}$ in water vapour was measured to assess the system capacity to resolve fine spectral signatures ${ }^{22}$. A sample gas, a mixture of water vapour ( partial pressure $=10 \mathrm{~Pa})$ and nitrogen $($ partial pressure $=320 \mathrm{~Pa})$, was introduced into the low-pressure gas cell. The sample has an expected pressure-broadening linewidth of $23 \mathrm{MHz}$ full-width at half-maximum (FWHM) from the self-broadening of water vapour, the collision-broadening induced by nitrogen ${ }^{21}$, and an expected Doppler-broadening linewidth of $0.026 \mathrm{MHz}^{23}$.

Figure 3(a) shows a power spectrum of the whole standard $\mathrm{THz}$ comb, obtained by taking the FT of the temporal waveform of 10 consecutive THz pulses $\left(N_{p}=10, t_{w}=N_{p} / f_{\text {rep } 1}=40 \mathrm{~ns}\right.$, number of signals averaged $=5000$, acquisition time $=1000 \mathrm{~s}$ ). The maximum dynamic range of $40 \mathrm{~dB}$ in power was achieved at $0.5 \mathrm{THz}$, and the observed spectral bandwidth was extended up to 2 THz. Figure 3(b) shows the amplitude spectrum of the standard $\mathrm{THz}$ comb expanded around $0.557 \mathrm{THz}$. The comb modes had a frequency gap of $250 \mathrm{MHz}$ and a linewidth of $25 \mathrm{MHz}$. This mode gap was exactly equal to $f_{\text {rep } 1}$ as shown in Fig. 1(b). On the other hand, the mode linewidth here was determined by the reciprocal of the temporal window $t_{w}(=40 \mathrm{~ns})$, although the intrinsic linewidth is as little as a few $\mathrm{Hz}^{24}$. The amplitude spectrum without incremental sweeping of the $\mathrm{THz}$ comb modes did not indicate the spectral shape of the absorption line due to the excessively coarse distribution of the comb modes compared with the absorption linewidth of the low-pressure water vapour.

Next, we demonstrated incremental sweeping of the comb modes across the absorption line at $0.557 \mathrm{THz}$ by changing the repetition frequencies of both lasers. Incremental increases of $f_{\text {rep } 1}$ and $f_{\text {rep } 2}$ $\left(=f_{\text {rep } 1}+f_{\text {offset }}\right)$ by $11,220.8 \mathrm{~Hz}\left(=\delta f_{\text {rep } 1}=\delta f_{\text {rep } 2}\right)$ were repeated ten times $\left(M=N_{p}=10\right)$ while keeping $f_{\text {offset }}$ at $50 \mathrm{~Hz}$. The resulting overlaid spectra of the comb modes are shown in Fig. 3(c). In this demonstration, a single shift of $f_{\text {rep } 1}$ by $0.004488 \%$ resulted in sweeping of the comb modes by $10 \%$ of their interval $(=25 \mathrm{MHz})$ due to the large number of THz comb modes $(m=2,228$ at $0.557 \mathrm{THz})$.
The frequency gaps between the comb modes in Fig. 3(b) were filled by interleaving additional frequency marks. In other words, a spectrally interleaved THz comb was successfully achieved. Although the comb mode amplitude is undulated due to a slight mismatching between the comb mode peaks and the spectral sampling points, a sharp spectral dip clearly appeared at the position of the water absorption line of $1_{10} \leftarrow 1_{01}$. The absorption was extracted from the power spectrum by taking the peak amplitude of each mode normalized with a reference spectrum obtained under identical conditions, as shown in Fig. 3(d). To determine the spectral linewidth, we fitted a Lorentzian function to the measured spectral profile because the spectral shape in $\mathrm{THz}$ region arises from the pressure broadening rather than Doppler broadening $(=0.026 \mathrm{MHz})^{23}$ at the present experimental condition. As a result, the spectral linewidth was determined to be $24 \mathrm{MHz}$, indicated by a red solid line in Fig. 3(d), and this value is consistent with the expected pressure broadening linewidth $(=23 \mathrm{MHz})$.

The same procedure was used to examine the $1_{10} \leftarrow 1_{01}$ water absorption line for various pressures. The black circles in Fig. 3(e) show FWHM of the observed absorption line with respect to the partial pressure of the water vapour, which was varied between $5 \mathrm{~Pa}$ and $160 \mathrm{~Pa}$. For comparison, the pressure broadening characteristic of this gas sample is expected from the self-broadening of water vapour and the collision broadening induced by nitrogen, as a red solid line in Fig. 3(e $)^{21}$. The experimental data were in good agreement with the expected line down to $25 \mathrm{MHz}$, and then deviated from it. This result clearly indicated that the increased spectral sampling density in the interleaved $\mathrm{THz}$ comb enhanced the spectral resolution of this gas spectroscopy to the level of the linewidth of the comb mode $(=25 \mathrm{MHz})$ rather than the gap size $(=250 \mathrm{MHz})$ for unswept measurements.

Spectroscopy of gas-phase acetonitrile. Since acetonitrile $\left(\mathrm{CH}_{3} \mathrm{CN}\right)$ is a very abundant species in the interstellar medium and is an ideal observational probe of the kinetic temperature and density of interstellar clouds ${ }^{25}$, we performed gas-phase spectroscopy of this molecule. Since $\mathrm{CH}_{3} \mathrm{CN}$ is a symmetric top molecule with a rotational constant $B$ of $9.2 \mathrm{GHz}$, it displays two features: a series of manifolds of rotational transitions regularly spaced by $2 \mathrm{~B}$ $(=18.4 \mathrm{GHz})$, and hyperfine structure of rotational transitions into each manifold determined by the centrifugal distortion constant 


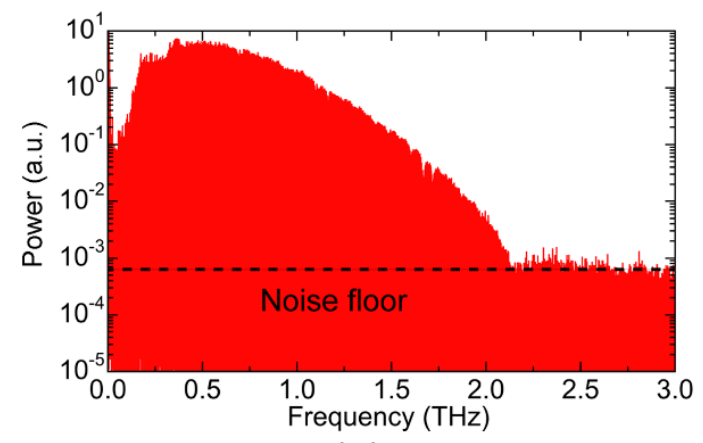

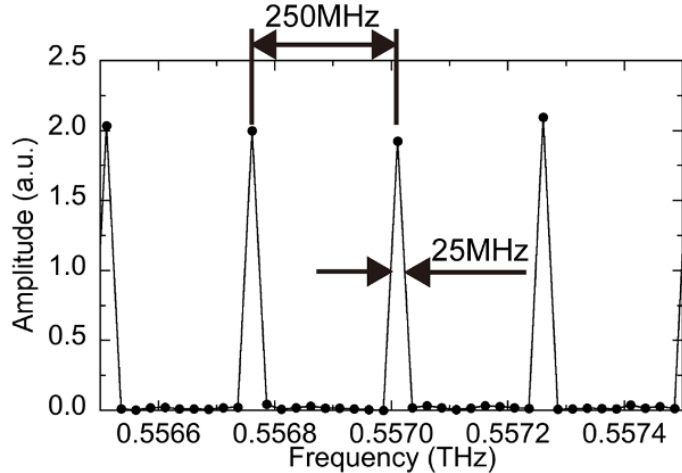

(b)

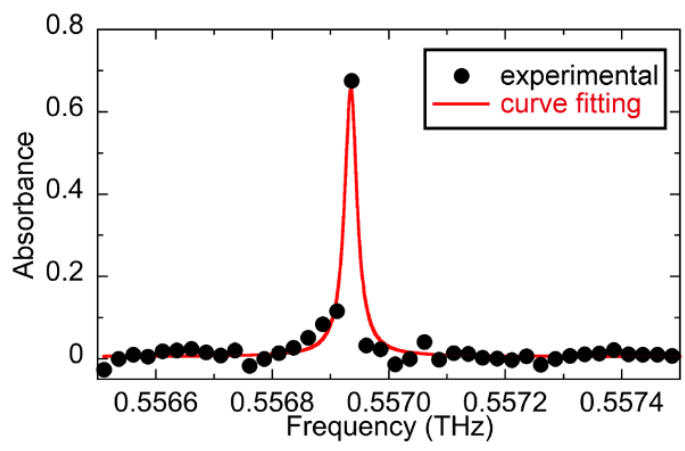

(d)

(a)

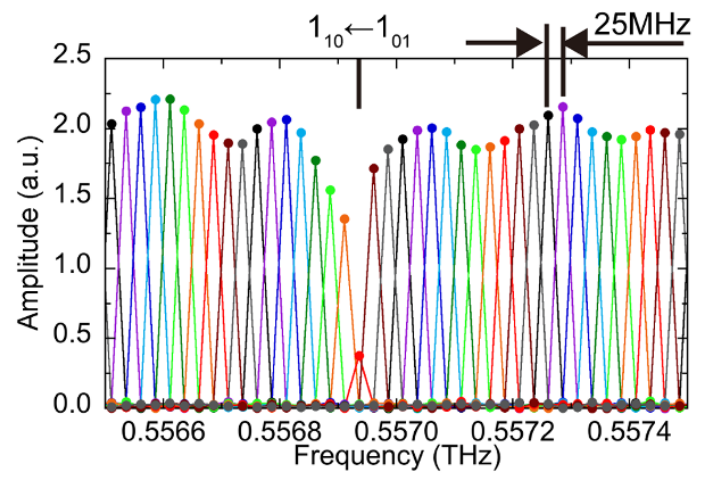

(c)

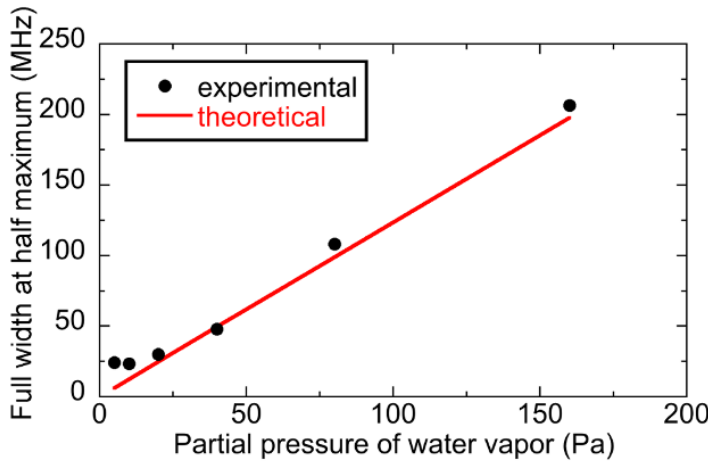

(e)

Figure $3 \mid$ (a) Power spectrum of the whole standard THz comb. Amplitude spectra of (b) standard $\mathrm{THz}$ comb and (c) interleaved THz comb around $0.557 \mathrm{THz}$ after passing through low-pressure water vapour contained in the gas cell. (d) Absorption spectrum and (e) pressure broadening characteristics of the rotational transition $1_{10} \leftarrow 1_{01}$ in water vapour.

$D_{J K}{ }^{25}$. Conventionally, it has been necessary to measure these two features separately by using broadband $\mathrm{THz}^{-\mathrm{TDS}^{26}}$ and a highresolution $\mathrm{CW}-\mathrm{THz}$ spectrometer ${ }^{27}$ due to the limited frequency resolution, accuracy, and/or coverage in traditional $\mathrm{THz}$ spectroscopy. Recently, one-pulse-period ASOPS-THz-TDS was used to observe both the manifold and the hyperfine-structure features simultaneously ${ }^{20}$. However, since its spectral resolution remained at the laser repetition frequency, it was still difficult to fully resolve all of the hyperfine structure. The reduced combmode linewidth and increased spectral sampling density offered by the spectrally interleaved dual-THz-comb configuration should allow the hyperfine structure to be resolved at high resolution and accuracy.

First, we performed spectroscopy of gas-phase $\mathrm{CH}_{3} \mathrm{CN}$ at $40 \mathrm{~Pa}$ enclosed in a gas cell by using the standard $\mathrm{THz}$ comb, obtained by taking the FT of the temporal waveform of 10 consecutive THz pulses $\left(N_{p}=10, t_{w}=N_{p} / f_{\text {rep } 1}=40 \mathrm{~ns}\right)$. Figure 4 (a) shows the absorption spectrum of this gas sample obtained by using the standard $\mathrm{THz}$ comb, in which a series of manifolds from $\mathrm{J}=16$ to $\mathrm{J}=55$ was clearly confirmed at intervals of $18.4 \mathrm{GHz}$ within a frequency range from 0.3 to $1.0 \mathrm{THz}$ in the same manner as the $\mathrm{THz}^{-\mathrm{TDS}^{26}}{ }^{2}$ Next, we used an interleaved $\mathrm{THz}$ comb with a mode linewidth of $25 \mathrm{MHz}$ for high-precision spectroscopy and allowed the hyperfine structure into a single manifold around $0.64 \mathrm{THz}(\mathrm{J}=35$ to $\mathrm{J}=34)$ to be observed, as shown in Fig. 4(b), in the same manner as the high-resolution CW$\mathrm{THz}$ spectrometer ${ }^{27}$. To assign these absorption lines, multi-peak fitting analysis based on a Lorentzian function was used, indicated by the red solid line in Fig. 4(b). In comparison with literature values reported in the JPL database (see blue dashed line) ${ }^{28}$, we successfully assigned lines $\mathrm{K}=2$ to 10 within a frequency discrepancy of $4.7 \pm$ $2.6 \mathrm{MHz}$ (mean \pm standard deviation for 9 absorption lines). To assign lines $\mathrm{K}=0$ and 1 with the frequency separation of $12 \mathrm{MHz}$, the linewidth and incremental step of the comb modes should be further reduced. To this end, we expanded the time window $t_{w}$ to $400 \mathrm{~ns}\left(=N_{p} / f_{\text {rep } 1}\right)$, in which the temporal waveform of 100 consecutive THz pulses was measured $\left(N_{p}=100\right)$. It should be emphasized that the time window of 400 ns corresponds to a mechanical timedelay scanning by $60 \mathrm{~m}$ when a traditional THz-TDS is used. Only ASOPS-THz-TDS can provide such an incomparably wide time window. This reduces the linewidth of the comb mode to $2.5 \mathrm{MHz}$. 


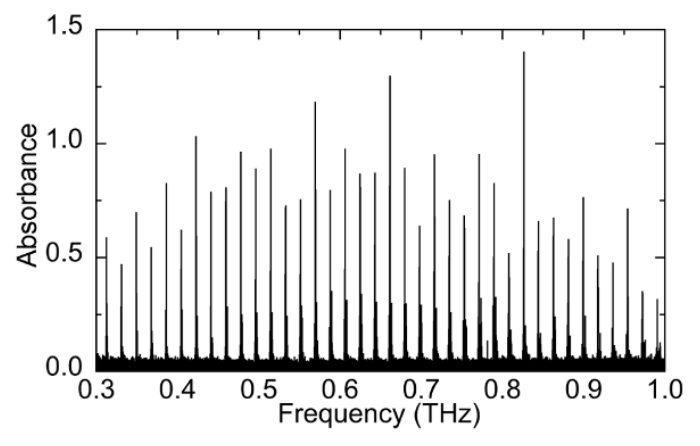

(a)

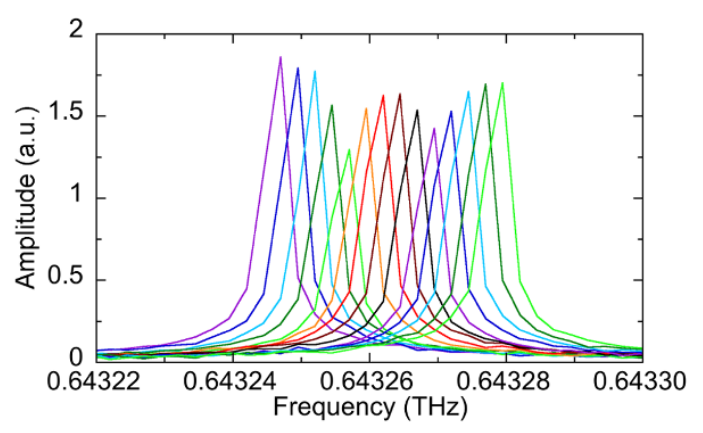

(c)

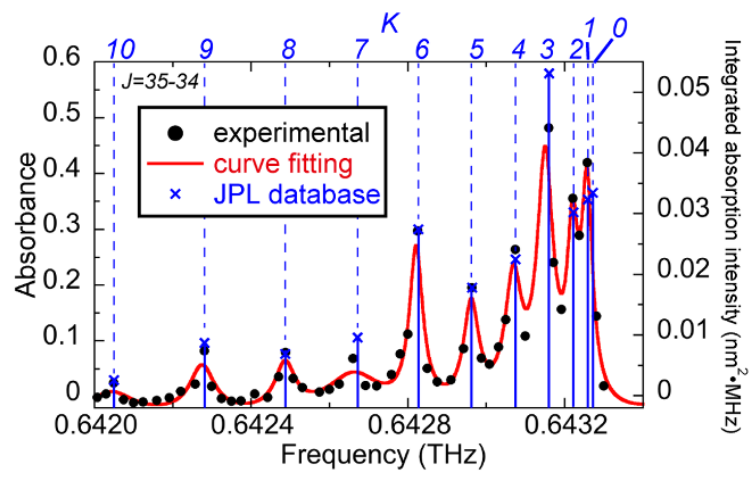

(b)

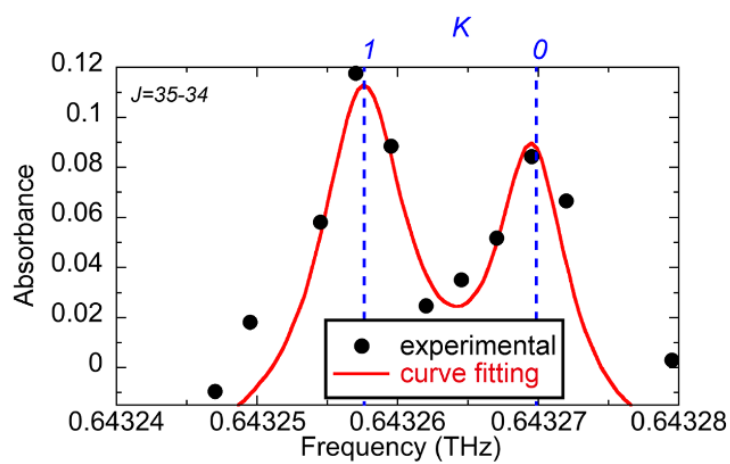

(d)

Figure 4 Absorption spectrum of low-pressure acetonitrile gas (a) within a frequency range from 0.3 to $1 \mathrm{THz}$ and (b) around $0.6428 \mathrm{THz}$. (c) Amplitude spectrum and (d) absorption spectrum obtained by 15 incremental sweeps of THz comb mode across two adjacent absorption peaks.

Incremental sweeping of this comb mode was repeated 15 times at intervals of $2.5 \mathrm{MHz}$ across the spectral region of the two absorption lines of $\mathrm{K}=0$ and 1 (gas pressure $=20 \mathrm{~Pa}$, pressure broadening linewidth $=5.9 \mathrm{MHz}$, Doppler broadening linewidth = $0.020 \mathrm{MHz})^{24,29}$. Figure 4(c) indicated that two spectral dips clearly appeared although the pressure broadening makes them overlap partially. The spectral linewidth was determined to be $7.5 \mathrm{MHz}$ for $\mathrm{K}=0$ and $6.1 \mathrm{MHz}$ for $\mathrm{K}=1$ by performing multi-peak fitting analysis, as shown in Fig. 4(d), which are consistent with the expected pressure broadening linewidth $(=5.9 \mathrm{MHz})$. These results clearly indicated that the reduced comb-mode linewidth and the increased spectral sampling density further enhanced the spectral resolution. We also determined their centre frequencies to be $0.643257578 \mathrm{THz}$ for $\mathrm{K}=0$ and $0.643269502 \mathrm{THz}$ for $\mathrm{K}=1$ from the multi-peak fitting analysis. The discrepancy of the centre frequencies from the literature values in the JPL database (see blue dashed line) ${ }^{28}$ was $0.578 \mathrm{MHz}$ for $\mathrm{K}=0$ and $0.502 \mathrm{MHz}$ for $\mathrm{K}=1$, corresponding to a mean spectral accuracy of $8.39 \times 10^{-7}$. Since these discrepancies were within the spectral sampling interval of $2.5 \mathrm{MHz}$, further reduction of the mode linewidth and incremental step will improve the spectral accuracy as well as the spectral resolution. These results clearly indicated the high potential of the spectrally interleaved dual-THz-comb spectroscopy to simultaneously probe multiple absorption lines of low-pressure molecular gas.

The accuracy of the absorption strength is also important to make a reliable quantitative analysis in gas-phase spectroscopy under low pressure. To evaluate the quality of the obtained spectrum, we made a simple comparison of our spectrum with the integrated absorption intensities in JPL database ${ }^{28}$, indicated as blue solid lines marked with blue cross in Fig. 4(b). The integrated absorption intensity is defined as an integration of the absorption spectrum and depends on both the absorption coefficient and linewidth. Since the pressure broadening coefficient of individual absorption line for this gas sample is unknown, this comparison gives the correctness for the relative variation between neighbouring lines rather than the correctness of the absolute value for absorbance. The relative variation is reasonably well reproduced and is limited by the signal-to-noise ratio of the measurements and the accuracy of the line strength calculations included in the database.

\section{Discussion}

The proposed method can enhance the spectral sampling interval to the level of the intrinsic linewidth of the comb mode (typically, less than a few $\mathrm{Hz})^{24}$ by increasing the observed time window of the $\mathrm{THz}$ pulse train and decreasing the incremental step of the comb sweeping, in principle. However, the actual linewidth in the comb-moderesolved spectrum may be limited by the timing jitter between the two fibre lasers. Here we discuss the contribution of the timing jitter to the linewidth of the comb mode. This contribution is estimated by the accumulated timing jitter per time increment at the end of the sample interval and the number of the comb modes because such the timing jitter causes fluctuation in the size of the time window and hence the frequency increment in the Fourier spectrum ${ }^{30}$, which leads to broadening of the comb mode linewidth. In the case of the spectrally interleaved $\mathrm{THz}$ comb with a mode linewidth of $2.5 \mathrm{MHz}$, the temporal waveform of 100 consecutive $\mathrm{THz}$ pulses was acquired. The accumulated timing jitter was 117 fs for the 100-th THz pulse when the temporal waveform was measured at a sampling interval of $25 \mathrm{fs}$; this contributes to a $1.75 \mathrm{kHz}$ increase in the mode linewidth at $0.6 \mathrm{THz}$. Therefore, we can conclude that the comb mode linewidth in the present system is not influenced by the timing jitter but is determined by the size of the time window; this is demonstrated by the absorption linewidth in the results shown in Fig. 4(d). This indicates that the present system has further linewidth gains that can be implemented. 
Table 1 | Spectroscopic performance of various $\mathrm{THz}$ spectrometers

Frequency coverage $(\mathrm{THz})$

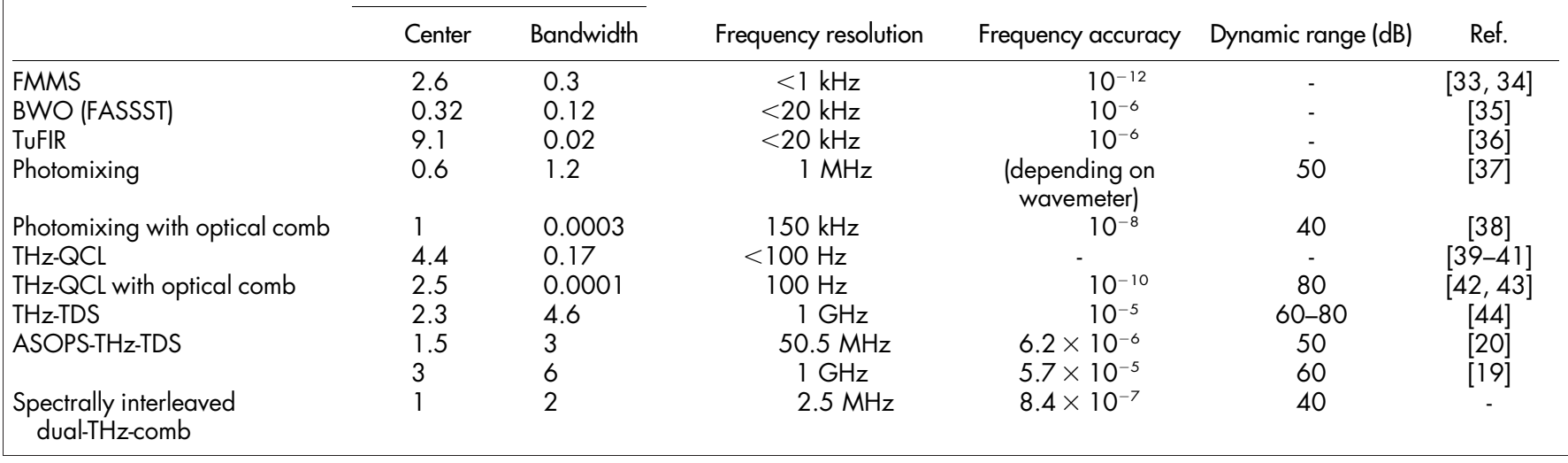

Next, we discuss the applicability of the spectral interleaving technique to an optical comb. In the case of a $\mathrm{THz}$ comb, the only way to achieve the spectrally interleaved comb is by sweeping $f_{\text {rep } 1}$ because the $\mathrm{THz}$ comb is a harmonic frequency comb of $f_{\text {rep } 1}$ and does not contain $f_{\text {ceo }}$. Sweeping of $f_{\text {rep } 1}$ has the advantage that a small increment of $f_{\text {rep } 1}$ causes a large change in the comb mode frequency due to the large number of comb modes. However, the resulting comb modes are not always interleaved appropriately over the full spectral range but are partially interleaved around the target frequency because the amount of frequency shift of each comb mode depends on its mode number $m$ as well as $\delta f_{\text {rep } 1}$ [see Fig. 1(c)]. For example, the comb gap is more densely interleaved below the target frequency whereas it is more coarsely interleaved over the target frequency. On the other hand, since the absolute frequency of the optical comb mode is given by $f_{c e o}+m f_{r e p 1}$, it can be spectrally interleaved by sweeping $f_{\text {ceo }}$ as well as $f_{\text {rep } 1}$. Although the previous studies of interleaved comb in optical region were based on sweeping of $f_{\text {rep } 1}^{13-16}$, sweeping of $f_{c e o}$ allows us to make the optical comb interleave uniformly over the full spectral range. One technical challenge of this method is large tuning of $f_{\text {ceo }}$ over $f_{\text {rep } 1} / 2$ in order to fully cover the frequency gap between comb modes. Extension of the fully interleaved technique to an optical comb will further expand the possible applications of the optical-comb-based, broadband spectroscopy due to the uniformly spectral sampling density.

\section{Conclusion}

To achieve a spectral sampling density equal to the linewidth of each comb mode, frequency gaps between $\mathrm{THz}$ comb modes were successfully interleaved by using swept dual $\mathrm{THz}$ combs. This is the first demonstration of overcoming the inherent limitation in $\mathrm{THz}$ comb, namely, the excessively discrete distribution of the comb modes limiting the fine spectral sampling for broadband spectroscopy. To demonstrate its superiority, we applied the interleaved $\mathrm{THz}$ comb to high-precision broadband spectroscopy of gas-phase molecules at low pressure. The resulting spectral resolution and accuracy were $2.5 \mathrm{MHz}$ and $8.39 \times 10^{-7}$, respectively. The proposed method has the potential to achieve the precise analysis of gas molecules in the atmospheric and space environments as well as combustion processes because the rotational transitions give them particularly rich spectral fingerprints in $\mathrm{THz}$ region while $\mathrm{THz}$ radiation is insensitive to scattering particles in optical region. Simultaneous achievement of high resolution, high accuracy, and broad spectrum by spectrally interleaved dual-THz-comb spectroscopy would enable us to discriminate densely distributed absorption lines correctly even though the target gases are mixed with aerosols, smoke, dust, clouds, or soot; for example, gas analysis in the smoke ${ }^{31}$ or sooty flame ${ }^{32}$.

\section{Methods}

Experimental setup. The individual repetition frequencies of the dual mode-locked Er-doped fibre lasers $\left(f_{\text {rep } 1}=250,000,000 \mathrm{~Hz}\right.$ and $f_{\text {rep } 2}=f_{\text {rep } 1}+f_{\text {offset }}=$ $250,000,050 \mathrm{~Hz})$ and the frequency offset between them $\left(f_{\text {offset }}=f_{\text {rep } 2}-f_{\text {rep } 1}=\right.$ $50 \mathrm{~Hz}$ ) were stabilized by two independent laser control systems referenced to a rubidium frequency standard (Rb-FS, accuracy $=5 \times 10^{-11}$, instability $=2 \times 10^{-11}$ at $1 \mathrm{~s}$ ), as shown in Fig. 2. Furthermore, $f_{\text {rep } 1}$ and $f_{\text {rep } 2}$ could be respectively tuned over a frequency range of $\pm 0.8 \%$ by changing the cavity length. After wavelength conversion of the two laser beams by second-harmonic generation (SHG) with nonlinear crystals, pulsed $\mathrm{THz}$ radiation was emitted from a dipole-shaped, low-temperature-grown (LTG), GaAs photoconductive antenna (PCA1) triggered by pump light $\left(\lambda_{c}=\right.$ $775 \mathrm{~nm}, \Delta \tau=80 \mathrm{fs}, P_{\text {mean }}=19 \mathrm{~mW}$ ), passed thorough a low-pressure gas cell (length $=500 \mathrm{~mm}$, diameter $=40 \mathrm{~mm}$ ), and was then detected by another dipoleshaped LTG GaAs photoconductive antenna (PCA2) triggered by probe light $\left(\lambda_{c}=\right.$ $\left.775 \mathrm{~nm}, \Delta \tau=80 \mathrm{fs}, P_{\text {mean }}=9 \mathrm{~mW}\right)$. The optical path, in which the $\mathrm{THz}$ beam propagated except for the part in the gas cell, was purged with dry nitrogen gas to avoid absorption by atmospheric moisture. A small portion of the output light from the two lasers were fed into a sum-frequency-generation cross-correlator (SFG-XC). The resulting SFG signal was used to generate a time origin signal in the ASOPS-THzTDS. After amplification with a current preamplifier (AMP, bandwidth $=1 \mathrm{MHz}$, gain $=4 \times 10^{6} \mathrm{~V} / \mathrm{A}$ ), the temporal waveform of the output current from PCA2 was acquired with a digitizer (sampling rate $=2 \times 10^{6}$ samples/s, resolution $=20$ bit) by using the SFG-XC's output as a trigger signal and the frequency standard's output as a clock signal. Then, the time scale of the observed signal was multiplied by a temporal magnification factor of $f_{\text {rep } 1} / f_{\text {offset }}(=250,000,000 / 50=5,000,000)^{18}$. This sampling rate and this temporal magnification factor enabled us to measure the temporal waveform of the pulsed $\mathrm{THz}$ electric field at a sampling interval of $100 \mathrm{fs}$.

Comparison with other $\mathrm{THz}$ spectrometers. To underline the principal advantage of the spectrally interleaved dual-THz-comb spectroscopy, we compared its basic performance with that of conventional spectrometers using tunable $\mathrm{CW}-\mathrm{THz}$ wave and broadband $\mathrm{THz}$ wave. Table 1 summarizes the comparison of the frequency coverage, frequency resolution, frequency accuracy, and dynamic range of power (DR) among them. CW-THz spectrometers using frequency-multiplied microwave sources (FMMS) can achieve the high spectral resolution and accuracy at high DR by referencing a microwave frequency standard whereas tuning range of a single FMMC is usually limited within 10 to $20 \%$ of a centre frequency ${ }^{33,34}$. CW-THz spectrometer with backward wave oscillator (BWO), also called to fast-scan submillimeter spectroscopy technique (FASSST), can extend the frequency coverage more widely while maintaining the similar resolution; however, its frequency accuracy is limited by the performance of Fabry-Perot cavity for frequency monitoring of $\mathrm{BWO}^{35}$. Although difference frequency mixing of $\mathrm{CO}_{2}$ laser and ${ }^{15} \mathrm{NH}_{3}$ laser with tunable microwave sideband generation (TuFIR) provides a spectral resolution below $20 \mathrm{kHz}$ for $\mathrm{CW}-\mathrm{THz}$ spectrometer, great care is required in obtaining precise frequency calibration and coherence ${ }^{36}$. Furthermore, in those CW-THz spectrometers above, it is difficult to fully cover the frequency range from $0.1 \mathrm{THz}$ to a few $\mathrm{THz}$ at once; it is necessary to select a suitable frequency multiplier chain, BWO tube, or laser emission frequency depending on the frequency band used, due to the limited tuning range ${ }^{33-36}$. Photomixing of tunable near-infrared lasers is a promising approach to achieve continuous tuning over $1 \mathrm{THz}$ while maintaining frequency resolution of $1 \mathrm{MHz}^{37}$. However, the frequency accuracy depends on the performance of a wavemeter that monitors the wavelength of near-infrared lasers. Although combination of the photomixing with optical comb makes the output frequency traceable to a microwave frequency standard, the continuous tuning range is largely reduced ${ }^{38}$. THz quantum cascade laser (THz-QCL) has the intrinsic characteristic of narrow linewidth ${ }^{39}$ as well as high output power ${ }^{40}$. Furthermore, introduction of external cavity configuration provided broad tunability to this source ${ }^{41}$; however, its continuously tuning range is 
limited within $10 \mathrm{GHz}$ whereas its discontinuous tuning range is achieved over $100 \mathrm{GHz}$. On the other hand, phase-locking of THz-QCL to the optical comb enables us to obtain the frequency accuracy traceable to a microwave frequency standard and the reduced linewidth ${ }^{42}$. However, the continuous tuning range is limited within $1 \mathrm{GHz}^{43}$.

In THz-TDS, the spectral resolution is determined by the length of mechanical time-delay scanning whereas the spectral accuracy depends on the positioning accuracy of a mechanical time-delay stage ${ }^{44}$. In ASOPS-THz-TDS, the spectral resolution depends on the laser repetition frequency: a lower repetition frequency leads to lower spectral resolution ${ }^{20}$ whereas a higher repetition frequency contributes to higher DR and wider spectral range ${ }^{19}$. On the other hand, since the spectral resolution of the spectrally interleaved dual-THz-comb spectroscopy depends on the comb mode linewidth, better spectral resolution can be achieved regardless of the repetition frequency. The frequency accuracy in ASOPS-THz-TDS and interleaved dual-THz-comb spectroscopy is determined by the spectral sampling interval and the stability of a temporal magnification factor of $f_{\text {rep } 1} / f_{\text {offset }}$, which is stabilized by the precise laser control. The high total balance of frequency resolution, frequency accuracy, and frequency coverage makes the interleaved dual-THz-comb spectroscopy more attractive than other THz spectrometers though its DR should be improved to widely apply it to various spectroscopic analyses.

1. Thorpe, M. J., Moll, K. D., Jones, R. J., Safdi, B. \& Ye, J. Broadband cavity ringdown spectroscopy for sensitive and rapid molecular detection. Science 311, 1595-1599 (2006).

2. Diddams, S. A., Hollberg, L. \& Mbele, V. Molecular fingerprinting with the resolved modes of a femtosecond laser frequency comb. Nature 445, 627-630 (2007).

3. Adler, F., Thorpe, M. J., Cossel, K. C. \& Ye, J. Cavity-enhanced direct frequency comb spectroscopy: technology and applications. Annu. Rev. Anal. Chem. 3, 175-205 (2010).

4. Mandon, J., Guelachvili, G. \& Picqué, N. Fourier transform spectroscopy with a laser frequency comb. Nature Photon. 3, 99-102 (2009).

5. Schiller, S. Spectrometry with frequency combs. Opt. Lett. 27, 766-768 (2002).

6. Keilmann, F., Gohle, C. \& Holzwarth, R. Time-domain mid-infrared frequencycomb spectrometer. Opt. Lett. 29, 1542-1544 (2004).

7. Yasui, T., Kabetani, Y., Saneyoshi, E., Yokoyama, S. \& Araki, T. Terahertz frequency comb by multifrequency-heterodyning photoconductive detection for high-accuracy, high-resolution terahertz spectroscopy. Appl. Phys. Lett. 88, 241104 (2006)

8. Bernhardt, B. et al. Cavity-enhanced dual-comb spectroscopy. Nature Photon. 4, 55-57 (2010).

9. Ideguchi, T., Poisson, A., Guelachvili, G., Hänsch, T. W. \& Picqué, N. Adaptive dual-comb spectroscopy in the green region. Opt. Lett. 37, 4847-4849 (2012).

10. Coddington, I., Swann, W. C. \& Newbury, N. R. Time-domain spectroscopy of molecular free-induction decay in the infrared. Opt. Lett. 35, 1395-1397 (2010).

11. Yasui, T. et al. Fiber-based, hybrid terahertz spectrometer using dual fibre combs. Opt. Lett. 35, 1689-1691 (2010).

12. Hsieh, Y.-D. et al. Terahertz comb spectroscopy traceable to microwave frequency standard. IEEE Trans. Terahertz Sci. Tech. 3, 322-330 (2013).

13. Jacquet, P. et al. Frequency comb Fourier transform spectroscopy with $\mathrm{kHz}$ optical resolution. Fourier Transform Spectroscopy 2009 (Vancouver, 2009), FMB2.

14. Baumann, E. et al. Spectroscopy of the methane $v_{3}$ band with an accurate midinfrared coherent dual-comb spectrometer. Phys. Rev. A 84, 062513 (2011).

15. Hipke, A., Meek, S. A., Guelachvili, G., Hänsch, T. W. \& Picque, N. Doppler-free broad spectral bandwidth two-photon spectroscopy with two laser frequency combs. CLEO: Science and Innovations 2013 (San Jose, 2013), CTh5C.

16. Kuse, N., Ozawa, A., Ito, I. \& Kobayashi, Y. Dual-comb saturated absorption spectroscopy. CLEO: Science and Innovations 2013 (San Jose, 2013), CTu2I.

17. Janke, C., Först, M., Nagel, M., Kurz, H. \& Bartels, A. Asynchronous optical sampling for high-speed characterization of integrated resonant terahertz sensors. Opt. Lett. 30, 1405-1407 (2005).

18. Yasui, T., Saneyoshi, E. \& Araki, T. Asynchronous optical sampling terahertz time-domain spectroscopy for ultrahigh spectral resolution and rapid data acquisition. Appl. Phys. Lett. 87, 061101 (2005).

19. Klatt, G., Gebs, R., Janke, C., Dekorsy, T. \& Bartels, A. Rapid-scanning terahertz precision spectrometer with more than $6 \mathrm{THz}$ spectral coverage. Opt. Express 17 , 22847-22854 (2009).

20. Yasui, T. et al. Enhancement of spectral resolution and accuracy in asynchronousoptical-sampling terahertz time-domain spectroscopy for low-pressure gas-phase analysis. Opt. Express 20, 15071-15078 (2012).

21. Seta, T. et al. Pressure broadening coefficients of the water vapour lines at 556.936 and 752.033 GHz. J. Quantum Spectrosc. Radiat. Transfer 109, 144-150 (2008).

22. Yasui, T. et al. Gapless THz comb spectroscopy. Imaging and Applied Optics Congress: Fourier Transform Spectroscopy 2013 (Arlington, 2013), FT1E.3.

23. Hollas, J. M. Modern Spectroscopy (John Wiley \& Sons, Chichester, 2003).
24. Yasui, T. et al. Real-time monitoring of continuous-wave terahertz radiation using a fiber-based, terahertz-comb-referenced spectrum analyzer. Opt. Express 17, 17034-17043 (2009).

25. Kessler, M., Ring, H., Trambarulo, R. \& Gordy, W. Microwave spectra and molecular structures of methyl cyanide and methyl isocyanide. Phys. Rev. 79, $54-56$ (1950).

26. Mittleman, D. M., Jacobsen, R. H., Neelamani, R., Baraniuk, R. G. \& Nuss, M. C. Gas sensing using terahertz time-domain spectroscopy. Appl. Phys. B 67, 379-390 (1998).

27. Matsuura, S. et al. High-resolution terahertz spectroscopy by a compact radiation source based on photomixing with diode lasers in a photoconductive antenna. J. Mol. Spectrosc. 187, 97-101 (1998).

28. Rothman, L. S. et al. Submillimeter, millimeter, and microwave spectral line catalog. J. Quant. Spectrosc. Radiat. Transf. 60, 883-890 (1998).

29. Pickett, H. M. et al. The HITRAN 2008 molecular spectroscopic database. J. Quant. Spectrosc. Radiat. Transf. 110, 533-572 (2009).

30. Bartels, A. et al. Ultrafast time-domain spectroscopy based on high-speed asynchronous optical sampling. Rev. Sci. Instrum. 78, 035107 (2007).

31. Bigourd, D. et al. Detection and quantification of multiple molecular species in mainstream cigarette smoke by continuous-wave terahertz spectroscopy. Opt. Lett. 31, 2356-2358 (2006).

32. Bassi, J., Stringer, M., Miles, B. \& Zhang, Y. Terahertz time-domain spectroscopy of high-pressure flames. Front. Energy Power Eng. China 3, 123-133 (2009).

33. Drouin, B. J., Maiwald, F. W. \& Pearson, J. C. Application of cascaded frequency multiplication to molecular spectroscopy. Rev. Sci. Instrum. 76, 093113 (2005).

34. Pearson, J. C. et al. Demonstration of a room temperature $2.48-2.75 \mathrm{THz}$ coherent spectroscopy source. Rev. Sci. Instrum. 82, 093105 (2011).

35. Mittleman, D. M. Sensing with THz radiation (Springer, Berlin, 2003).

36. Odashima, H., Zink, L. R. \& Evenson, K. M. Tunable far-infrared spectroscopy extended to 9.1THz. Opt. Lett. 24, 406-407 (1999).

37. Deninger, A. J. et al. Precisely tunable continuous-wave terahertz source with interferometric frequency control. Rev. Sci. Instrum. 79, 044702 (2008).

38. Hindle, F. et al. Widely tunable THz synthesizer. Appl. Phys. B 104, 763-768 (2011).

39. Vitiello, M. S. et al. Quantum-limited frequency fluctuations in a terahertz laser. Nature Photon. 6, 525-528 (2012).

40. Williams, B. S., Kumar, S., Hu, Q. \& Reno, J. L. High-power terahertz quantumcascade lasers. Electron. Lett. 42, 89-90 (2006).

41. Lee, A. W.-M., Williams, B. S., Kumar, S., Hu, Q. \& Reno, J. L. Tunable terahertz quantum cascade lasers with external gratings. Opt. Lett. 35, 910-912 (2010).

42. Barbieri, S. et al. Phase-locking of a $2.7-\mathrm{THz}$ quantum cascade laser to a modelocked erbium-doped fibre laser. Nature Photon. 4, 636-640 (2010).

43. Consolino, L. et al. THz spectroscopy with an absolute frequency scale by a QCL phase-locked to a THz frequency comb. 38th International Conference on Infrared, Millimeter, and Terahertz Waves (Mainz, 2013), We10-5.

44. Hoshina, H. et al. Precise measurement of pressure broadening parameters for water vapor with a terahertz time-domain spectrometer. J. Quant. Spectrosc. Radiat. Transfer 109, 2303-2314 (2008)

\section{Acknowledgments}

This work was supported by Collaborative Research Based on Industrial Demand from the Japan Science and Technology Agency. We also gratefully acknowledge financial support from the Renovation Centre of Instruments for Science Education and Technology at Osaka University, Japan. The authors are grateful to Dr. M. Hashimoto of Osaka University, Japan, and Dr. H. Hoshina of RIKEN, Japan, for fruitful discussions.

\section{Author contributions}

Y.-D.H. and T.Y. conceived the idea and wrote the paper. Y.-D.H., Y.I., Y.S. and F.H. built the instrument, performed the experiment, and analysed data. S.Y. conceived the idea and contributed to manuscript preparation. H.I., K.M., T.A. and T.Y. contributed to manuscript preparation and supervised the research.

\section{Additional information}

Competing financial interests: The authors declare no competing financial interests.

How to cite this article: Hsieh, Y.-D. et al. Spectrally interleaved, comb-mode-resolved spectroscopy using swept dual terahertz combs. Sci. Rep. 4, 3816; DOI:10.1038/srep03816 (2014).

(c) (1) () $\odot$ This work is licensed under a Creative Commons Attribution-

BY NC No Nommercial-NoDerivs 3.0 Unported license. To view a copy of this license, visit http://creativecommons.org/licenses/by-nc-nd/3.0 